\title{
Symptomatic bilateral extra-cranial vertebral artery dissections induced by sexual activity
}

\author{
Casey Michael ${ }^{1}$, Whaley Calvin ${ }^{1}$, Hawkins Christopher ${ }^{1}$, Ajeet Gordhan ${ }^{2}$ \\ 1. Department of Neurosurgery, Advocate Bromenn Medical Center, IL, USA. 2. Department of Interventional \\ Neuroradiology, OSF St J oseph's Medical Center, IL, USA
}

Correspondence: Ajeet Gordhan. Address: Department of Interventional Neuroradiology, OSF St Joseph's Medical Center, 2200 East Washington St, Bloomington, IL, USA. Email: agordhan@hotmail.com

Received: December 1, 2015

Accepted: January 12, $2016 \quad$ Online Published: February 1, 2016

DOI : $10.5430 /$ ijdi.v3n1p72

URL: http://dx.doi.org/10.5430/ijdi.v3n1p72

\section{Abstract}

Introduction: Trauma is a well-recognized cause of vertebral artery dissection (VAD) and even minor episodes of trauma, including sneezing and vigorous exercise, have been implicated. Symptomatic bilateral vertebral artery dissections after sexual activity to our knowledge has not been reported.

Case report: A 32-year-old female presented with a three week history of occipito-cervical headaches. Noninvasive imaging identified bilateral vertebral artery dissections. The etiology was inferred after a directed clinical history revealed sexual activity characterized by aggressive repeated neck hyperextension from hair-pulling during intercourse.

Conclusion: We report a case of symptomatic bilateral VAD in the setting sexual intercourse related trauma. The quality of clinical data gathering, in particular pertinent history taking is imperative in determining a potential cause. This is important in patient counselling so as to prevent recurrence.

\section{Keywords}

Traumatic extra-cranial vertebral artery dissection, Hyperextension

\section{I ntroduction}

Extra-cranial vertebral artery dissection (VAD) refers to a disruption of the structural integrity of the vertebral artery wall and is most commonly induced by trauma. This can lead to a series of clinical sequelae including headache, neck pain, and focal neurologic deficits. We report a case of bilateral VAD in the setting sexual intercourse related trauma, to our knowledge not previously described.

\section{Case report}

We present a 32-year-old female with three week history of dull, aching, posterior cervical/occipital headaches. In the ten days prior to presentation, the patient reported episodes of slurred speech, unsteady gait and blurred vision. The patient initially denied any traumatic event preceding her presentation. She had no family history of connective tissue disorders or arterial dissections. Clinically, she did not have a Marfanoid appearance. Her neurological and physical examination were significant for bilateral paraspinal muscle tenderness at C1-C2, dysmetria and dysdiokinesis. No nystagmus or significant 
gait instability was identified. The sensory and motor evaluation was unremarkable. Her ESR was not elevated and no serologic markers for a vasculitides was identified.

An MRI of the brain identified multiple bilateral cerebellar acute embolic infarcts (see Figure 1). For more specific evaluation of the vertebrobasilar arterial system in lieu of this finding, computerized tomographic angiography (CTA) was favored over ultrasound. The CTA identified diminution in caliber of the vertebral arteries bilaterally (see Figure 2). To differentiate congenital hypoplasia of the vertebral arteries from dissections, Magnetic resonance angiography (MRA) was performed. The MRA head and neck imaging study with fat suppression and 3D reformations demonstrated bilateral subacute mural hematomas of the V3 and V4 segments of the vertebral arteries bilaterally, confirming dissections (see Figure 3).

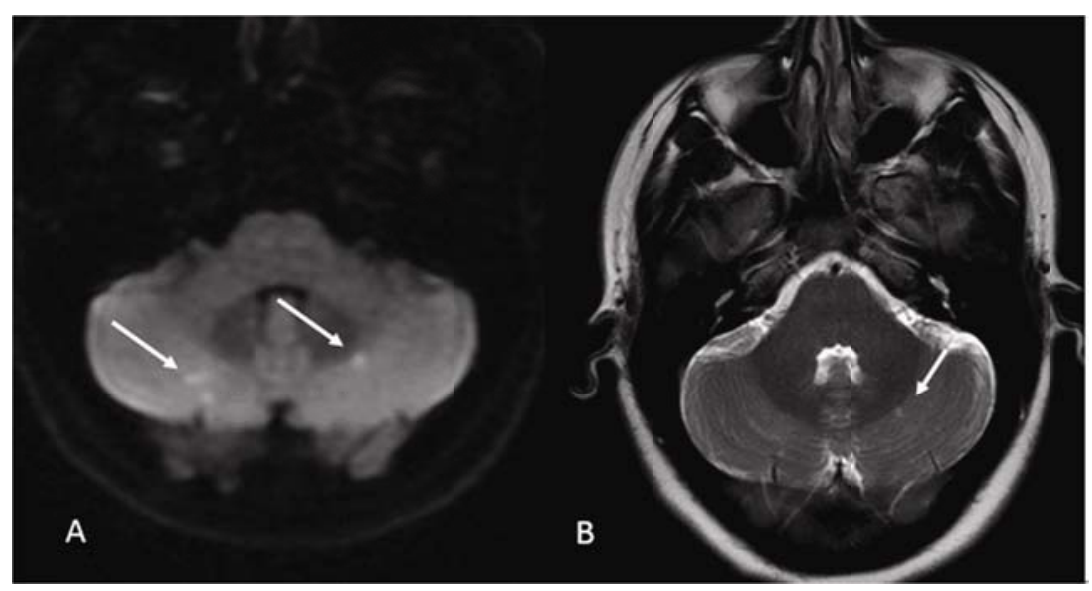

Figure 1. Axial select MRI diffusion (A) and T2 (B) sequence images through the posterior fossa demonstrating multiple embolic infarcts within the bilateral cerebellar hemispheres (arrows)

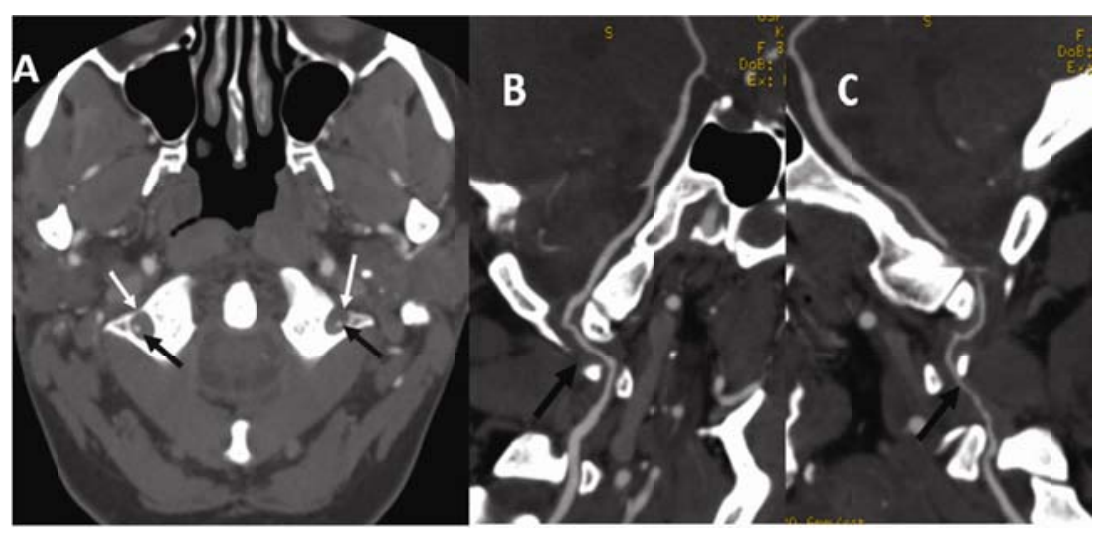

Figure 2. A: Maximum intensity projection CT angiography in the axial plane demonstrating diminutive caliber of the extra-cranial vertebral arteries bilaterally (black arrows) suspicious for dissections. Disproportionate size of the osseous transverse foramina relative to vessel size at the C1 ring (white arrows); B and C: Curved planar reformat CT angiography in the coronal plane demonstrating luminal irregularity of the extra-cranial vertebral arteries bilaterally (black thick arrows) compatible with dissections

A more detailed comprehensive clinical history interview to ascertain a cause was conducted, with further negative investigations for an underlying heritable connective tissue disorder and polycystic kidney disease. She denied chiropractic manipulation and had no history of migraine. The only trauma she described was repeated episodes of aggressive neck hyperextension with hair pulling during intercourse, just preceding her clinical symptoms. Medical management with Aspirin was initiated. She was counselled on sexual behavior modification to prevent a recurrence. Follow up CT angiography at 3 months revealed resolution of the dissections with compliance to sexual behavior modification (see Figure 4).

Published by Sciedu Press 


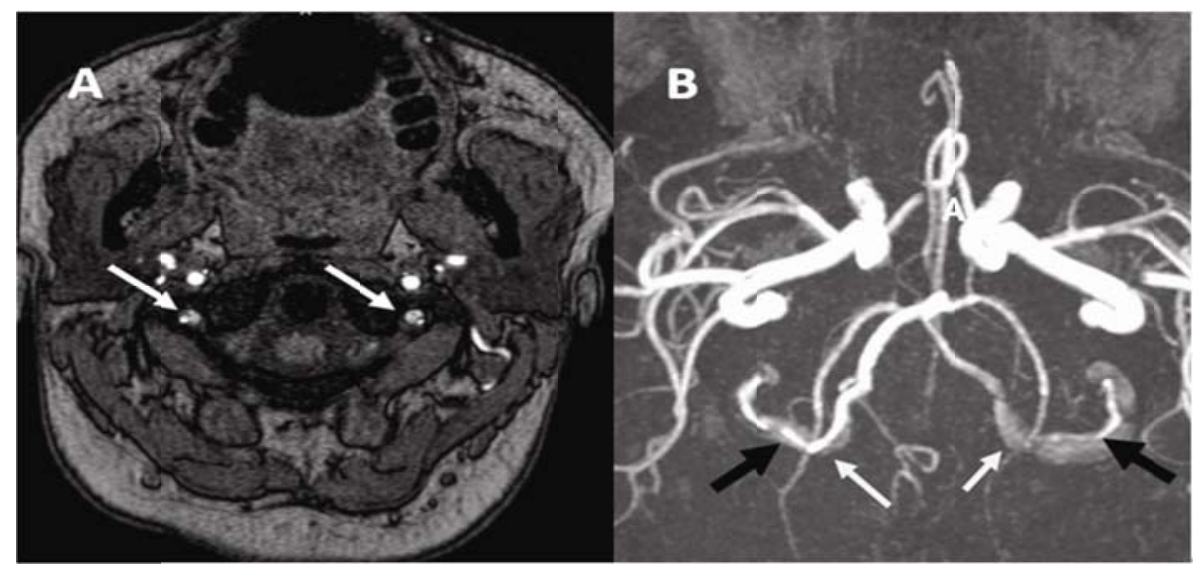

Figure 3. A: Fat suppressed select axial T1 weighted MRA in the axial plane confirming sub-acute hyper-intense mural hematoma (white thin arrows) contiguous to the diminutive bilateral vertebral arteries; B: 3D Time of flight MR angiography in the axial plane demonstrating luminal irregularity of the extra-cranial vertebral arteries bilaterally (black thick arrows) compatible with dissections. Associated T1 shine through from mural hematoma (white thin arrows)

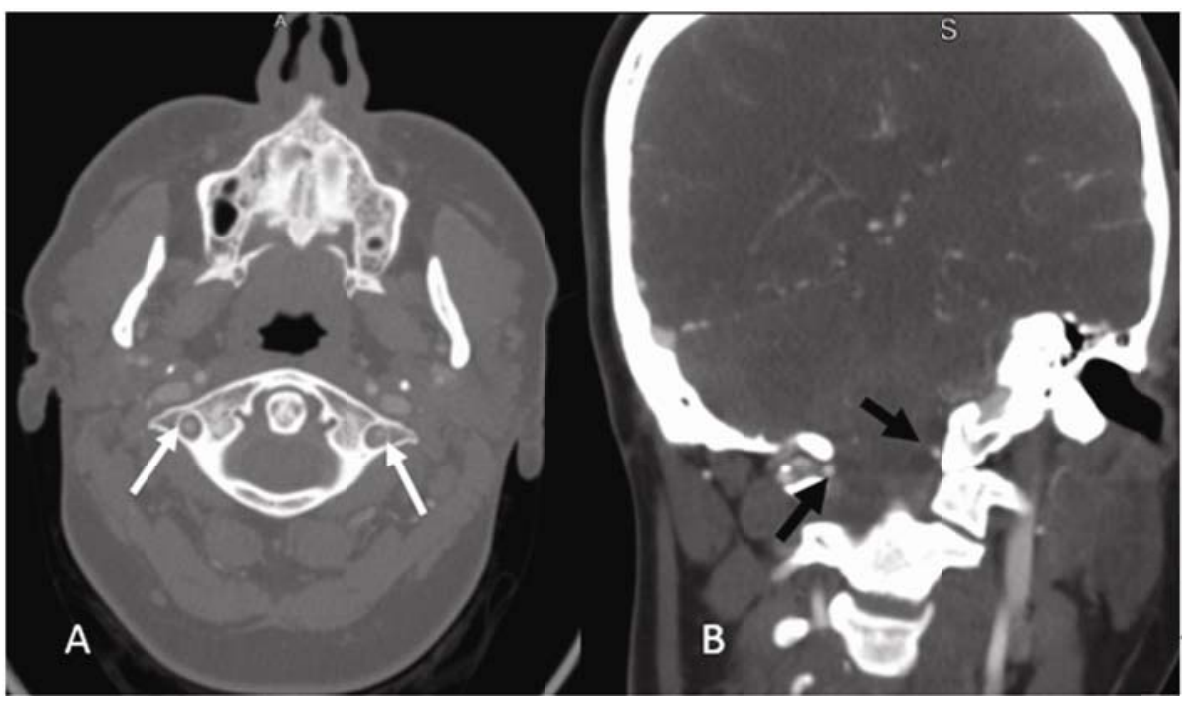

Figure 4. Select axial and oblique coronal CTA image demonstrating resolved dissections of the bilateral distal cervical vertebral arteries, characterized by significant improvement in vessel caliber (arrows)

\section{Discussion}

Spontaneous dissection of the vertebral artery is relatively uncommon, with an overall incidence of $1-1.5$ per $100,000{ }^{[1]}$. VAD is most common between the ages of 35 and $50^{[2]}$. Men and women tend to be affected in equal proportions, but women present on average five years younger than men ${ }^{[2]}$. Despite its relative rarity, spontaneous VAD, combined with carotid artery dissection, are estimated to cause $20 \%$ of strokes in patients younger than forty-five ${ }^{[3]}$. $10 \%-20 \%$ of traumatic cerebrovascular injury occur in the cervical region ${ }^{[4,5]}$. Furthermore, ischemia related to this type of injury is reported to be as high as $25 \%-58 \%{ }^{[4,5]}$.

VAD occurs when a tear in one or more of the arterial layers allows blood to separate the layers and fill the newly created space. This false lumen can cause vertebral artery stenosis when an intramural hematoma forms between the intima and media. When the hematoma is between the media and adventitia, the artery becomes susceptible to pseudoaneurysm formation. It is controversial whether the dissection originates from a direct intimal tear or rupture within the connective tissue and vaso vasorum of the tunica media ${ }^{[6]}$. VAD can present as an ischemic stroke either from local thrombus 
formation and subsequent hemodynamic failure or from embolization of the thrombus to a distant intracranial arterial site. Intra-dural penetration of a vertebral artery dissecting psedoaneurysm may result in subarachnoid hemorrhage ${ }^{[7]}$.

VAD are classified based on their location: intracranial or extra-cranial. Extra-cranial dissections tend to be more common. This has been attributed to the increased mobility of the extra-cranial segments, which are also vulnerable to injury from bony elements such as the vertebrae or styloid processes ${ }^{[8]}$. Vertebral artery dissection most commonly affects the V3 or V1 segments at the $\mathrm{C} 1$ to $\mathrm{C} 2$ level ${ }^{[9]}$. Intracranial dissections, while less common, tend to carry a poorer prognosis.

Minor trauma including sporting events, scuba diving, dancing, vigorous exercise, trampoline use, roller coaster rides, child birth, sneezing, and sexual intercourse have been described in the etiology of VADs ${ }^{[9]}$. Our patient's injuries were likely secondary to extreme hyperextension. Parent et al. described prolonged hyperextension of the neck while painting a ceiling as a mechanism of vertebral artery injury at the third vertebral artery segment ${ }^{[10]}$. Symptomatic bilateral extracranial vertebral artery dissections secondary to neck hyper-extension with traction during sexual intercourse has not been reported in the literature

\section{Conclusion}

Traumatic bilateral extra-cranial VAD by aggressive voluntary hyperextension related to intercourse has never been published. The mechanism of injury in this case report is novel and prompts the treating physician to interview the patient carefully and understand the benefits and limitations of different imaging modalities to confirm the diagnosis.

\section{Conflict of interests}

None for all authors.

\section{Acknowledgements}

Sunil Chauhan, MD, Department of Neurology, Advocate BroMenn Medical Center 1304 Franklin Ave, IL, USA.

Emilio Nardone, MD, Department of Neurosurgery, Advocate BroMenn Medical Center 1304 Franklin Ave, IL, USA.

\section{References}

[1] Bogousslavsky J, Regli F. Ischemic stroke in adults younger than 30 years of age. Cause and prognosis. Arch Neurol. 1987; 44: 479-482. PMid:3579657 http://dx.doi.org/10.1001/archneur.1987.00520170009012

[2] Schievink WI, Mokri B, O’Fallon WM. Recurrent spontaneous cervical-artery dissection. N Engl J Med. 1994; 330: 393-397. PMid:8284004 http://dx.doi.org/10.1056/NEJM199402103300604

[3] Bogousslavsky J, Pierre P. Ischaemic stroke in patients under age 45. Neurol Clin. 1992; 10: 113-124. PMid:1556998

[4] Fusco MR, Harrigan MR. Cerebrovascular dissections - a review part I: spontaneous dissections. Neurosurgery. 2011; 68(1): 242-257. PMid:21150764 http://dx.doi.org/10.1227/NEU.0b013e3182012323

[5] Fusco MR, Harrigan MR. Cerebrovascular dissections-a review part II: blunt cerebrovascular injury. Neurosurgery. 2011; 68(2): 517-530. PMid:21135751 http://dx.doi.org/10.1227/NEU.0b013e3181fe2fda

[6] Thanvi B, Munshi SK, Dawson SL, et al. Carotid and vertebral artery dissection syndromes. Postgrad Med J. 2005; 81: 383-388. PMid:15937204 http://dx.doi.org/10.1136/pgmj.2003.016774

[7] Caplan LR, Tettenborn B. Vertebraobasilar occlusive disease: review of selected aspects: I. Spontaneous dissection of extracranial and intracranial posterior circulation arteries. Cerebrovasc Dis. 1992; 2: 256-65. http://dx.doi.org/10.1159/000109025

[8] Chaves C, Estol C, Esnaola MM, et al. Spontaneous intracranial internal carotid artery dissection: report of 10 patients. Arch Neurol. 2002; 59: 977. PMid:12056934 http://dx.doi.org/10.1001/archneur.59.6.977

[9] Engelter ST, Grond-Ginsbach C, Metso TM, et al. Cervical artery dissection: trauma and other potential mechanical trigger events. Neurology. 2013; 80: 1950. PMid:23635964 http://dx.doi.org/10.1212/WNL.0b013e318293e2eb

[10] Parent AD, Harkey HL, Touchstone DA, et al. Lateral cervical spine dislocation and vertebral artery injury. Neurosurgery. 1992; 31(3): 501-509. PMid:1407430 http://dx.doi.org/10.1227/00006123-199209000-00012 